\title{
L'école algérienne au prisme des langues de scolarisation
}

Schooling in Algeria through the prism of languages of instruction

La escuela argelina en el prisma de las lenguas de escolarización

Khaoula Taleb Ibrahimi

\section{OpenEdition}

Journals

Édition électronique

URL : https://journals.openedition.org/ries/4493

DOI : $10.4000 /$ ries. 4493

ISSN : 2261-4265

Éditeur

France Education international

Édition imprimée

Date de publication : 1 décembre 2015

Pagination : 53-63

ISSN : 1254-4590

Référence électronique

Khaoula Taleb Ibrahimi, «L'école algérienne au prisme des langues de scolarisation », Revue internationale d'éducation de Sèvres [En ligne], 70 | décembre 2015, mis en ligne le 01 décembre 2017, consulté le 01 juillet 2021. URL : http://journals.openedition.org/ries/4493 ; DOI : https://doi.org/ $10.4000 /$ ries. 4493

() Tous droits réservés 


\title{
L'école algérienne au prisme des langues de scolarisation
}

\author{
Khaoula Taleb Ibrahimi \\ Université d'Alger 2
}

Il peut paraître paradoxal, eu égard à l'effort colossal déployé par l'Algérie depuis son accès à l'indépendance en 1962 pour assurer une scolarisation massive et ouverte à tous les petits Algériens ${ }^{1}$, que les performances de l'école algérienne soient aussi médiocres. L'indicateur le plus éloquent de cette faiblesse des performances est le taux de réussite à l'examen qui clôt la formation scolaire, le baccalauréat, qui fluctue pour le plus élevé de $59 \%$ à environ $40 \%$ pour le moins élevé, sur les deux dernières décennies. Sur 100 élèves qui entrent à l'école, seule la moitié arrive à boucler son cursus scolaire. Ces bacheliers, toutes filières confondues, entrent à l'université avec des difficultés notoires d'expression écrite en langue arabe, langue première de scolarisation, et des lacunes immenses dans la maîtrise de la langue française, première langue étrangère enseignée à l'école mais langue d'enseignement des disciplines scientifiques et technologiques à l'université. Sans vouloir occulter les nombreux autres facteurs ${ }^{2}$ qui pourraient expliquer ce constat, il reste que la problématique des langues de scolarisation, de leur maîtrise en tant que langues enseignées et de leur implication dans l'apprentissage des autres disciplines constitue le nœud gordien qui focalise l'intérêt des spécialistes mais aussi de l'opinion publique, tant la question des langues est éminemment politique. Le choix de la ou des langue(s) d'enseignement reste une question centrale pour les décideurs et pour les différents acteurs sociaux concernés : élites, familles, médias. Traversé par une constante têtue, celle de l'affirmation identitaire, ce choix a suscité, à chaque étape de sa mise en œuvre, des controverses passionnées : certaines questions fondamentales liées à l'histoire et au devenir de la nation ne sont toujours pas réglées.

En 1962, le jeune État indépendant se devait de relever le défi de réussir la première rentrée scolaire de la jeune République en répondant à l'espoir des familles de voir des centaines de milliers de petits Algériens franchir, pour la première fois, les portes d'un établissement scolaire; mais il lui fallait aussi assurer le passage le plus serein possible d'une école pensée par la puissance

1. Les chiffres de la rentrée de septembre 2015 sont éloquents : 8112475 élèves encadrés par 400000 enseignants. Avec 746643907000 dinars algériens, le budget de l’Éducation est le deuxième budget le plus important, après celui de la Défense.

2. La surcharge des classes, l'inégale répartition géographique de l'encadrement et la faiblesse de son niveau de formation. 
coloniale à une école algérienne qui permette d'asseoir une souveraineté retrouvée et tous les attributs de l'identité nationale. Et, dans le même temps, poser les jalons d'une école moderne qui ferait accéder les générations de l'indépendance au savoir, afin d'assurer la modernisation de leur société.

Nous allons dérouler le fil du passage d'une école exclusivement francophone, dans laquelle la langue arabe était enseignée uniquement en tant que langue, à une école exclusivement arabophone, dans laquelle la langue française n'est plus désormais enseignée qu'en tant que première langue étrangère, en passant par une phase intermédiaire de transition de quelques années, celle d'une école bilingue, dans laquelle les deux langues étaient les langues de scolarisation. Dans ce cheminement, l'État est l'acteur principal alors même que, à presque toutes les étapes du processus, il s'est vu chahuter par des résistances d'acteurs sociaux essentiellement privés.

\section{2-1965 : LES DÉBUTS DE L’ÉCOLE ALGÉRIENNE}

Au cours des trois premières années de l'indépendance, tout en maintenant le mode de structuration de l'école hérité de l'époque coloniale, des décisions sont prises pour redonner à la langue arabe la place qui doit être la sienne, consacrée par la Constitution comme seule langue nationale ${ }^{3}$ et officielle du pays. Elle est désormais enseignée à l'école sans toutefois être la langue de scolarisation. Il n'était pas possible en effet de couvrir les besoins en enseignants capables non seulement d'enseigner la langue mais surtout d'enseigner les autres disciplines dans cette langue. Face à ce déficit en encadrement ${ }^{4}$, il fallut faire appel tout à la fois à l'ancienne puissance coloniale et à des pays arabes frères pour l'envoi d'enseignants coopérants. Des centaines d'enseignants français, égyptiens, syriens et irakiens ont soutenu l'effort énorme de scolarisation de l'Algérie. Cette double coopération va contribuer à asseoir dans l'opinion une image contrastée des deux catégories de coopérants issus de France et des pays arabes, et par là même la dualité des représentations opposant les deux formations culturelles, les arabisants et les francisants, dualité qui a, jusqu'à aujourd'hui, empêché la constitution d'une intelligentsia nationale et fragmenté durablement l'espace culturel du pays. Cette fragmentation est à relier aux représentations contrastées des deux langues dans la société ${ }^{5}$. À cette époque, les différentes institutions scolaires françaises sont maintenues dans les grandes villes, accueillant

\footnotetext{
3. À tel point que cette appellation va supplanter la dénomination « langue arabe ».

4. Le premier recensement de la population, réalisé en 1966, le seul qui fasse référence à la formation en langues, le montre bien.

5. Nous avons amplement développé cette question dans notre ouvrage paru en 1995, ainsi que dans l'article paru dans Maghreb/Machrek de la même année.
} 
les enfants de toutes nationalités. Par ailleurs, certaines institutions religieuses chrétiennes continuent à posséder des établissements d'enseignement ouverts aux enfants algériens sans distinction de confession. Mais le processus d'arabisation compris comme le recouvrement par la langue arabe de sa place entière dans l'État, l'École et la société va s'intensifier et s'accélérer avec l'avènement de Houari Boumediene en 1965 et changer durablement la configuration scolaire du pays.

\section{5-1976 : ARABISATION ET ALGÉRIANISATION PROGRESSIVE DE L’ÉCOLE}

C'est au cours de cette décennie que vont être promulgués presque tous les textes régissant la politique d'arabisation de plusieurs secteurs de l'État, de l'administration et de l'école. Sans entrer dans le détail de cet arsenal juridique (voir Taleb Ibrahimi, 1995), nous nous contenterons de citer quelques décisions emblématiques : ouverture à la faculté de droit d'une filière en langue arabe en 1967 ; promulgation de l'ordonnance de 1968 qui rend obligatoire la connaissance de la langue arabe pour tous les fonctionnaires. En 1971, les enseignements d'histoire et de philosophie dans les filières littéraires au lycée sont complètement arabisés. En 1976, la promulgation de la Charte nationale vient consacrer les trois principes de la politique algérienne dans tous les domaines : démocratisation, arabisation, algérianisation. Ils constituent le socle de l'École fondamentale qui devait être mise en place dès la rentrée de 1976.

Ceci étant, entre 1965 et 1976, l'école algérienne a fonctionné d'une manière duale. Dans le primaire, si les trois premières années étaient complètement arabisées (la langue arabe est enseignée en tant que langue et est également la langue de scolarisation et d'enseignement des autres savoirs), les trois années suivantes se distribuent en fonction des deux langues d'enseignement (la langue arabe et la langue française introduite en tant que langue enseignée en troisième année primaire) selon le schéma suivant : un tiers des classes étaient entièrement arabisées et les deux tiers restants étaient bilingues, c'est-à-dire que toutes les matières scientifiques y étaient enseignées en français. Au collège, c'était quasiment le même schéma. Au lycée, l'arabisation va suivre le même cheminement, avec deux cursus complètement arabisés, à l'exception des mathématiques et de la géographie dans les filières littéraires, et selon le schéma des deux tiers dans les filières scientifiques. Toutefois, ce schéma des "deux tiers » n'a pu être maintenu en l'état, influencé qu'il était par les fluctuations des relations franco-algériennes et limité de plus en plus par l'insuffisance de l'encadrement francophone algérien, alors que la formation du corps enseignant en langue arabe s'est, elle, vu renforcée par la création des instituts technologiques de l'éducation. Le ministère a donc procédé à une plus large arabisation dépassant le tiers initialement prévu. 


\section{6-1989 : L'AFFIRMATION IDENTITAIRE ET L'AVÈNEMENT DE L'ÉCOLE FONDAMENTALE}

En 1976, le ministre en charge de l'Éducation nationale, Abdelkrim Benmahmoud, présente le projet de réforme du système éducatif dans le cadre de l'école fondamentale ${ }^{6}$, enseignement général s'étendant sur neuf années, avec trois paliers de trois années, et reposant sur les trois principes de l'algérianisation, avec un recours exclusif au personnel algérien, l'orientation technologique de la formation et enfin, l'arabisation, avec l'utilisation exclusive de la langue arabe comme langue de scolarisation et l'introduction d'une langue étrangère obligatoire, le français, dès la quatrième année d'études. En 1979, l'école fondamentale est instaurée, le processus d'arabisation totale est achevé pour les trois paliers en 1986 et, à la rentrée 1988-1989, l'enseignement secondaire est complètement arabisé.

Dans ce schéma, aucune mention n'est faite des langues maternelles, les dialectes arabes et berbères. Ces derniers sont complètement ignorés : les premiers sont stigmatisés parce qu'incorrects, le rôle de l'école, clairement affirmé, étant de veiller à la correction de la langue de la société à travers celle des écoliers. La langue française, elle, bénéficie d'un enseignement de douze années, sans pour autant que les élèves puissent maîtriser les compétences fondamentales, tant la formation des formateurs pèche par sa faiblesse et son incohérence eu égard à l'évolution des pratiques langagières des locuteurs algériens. De plus, l'ambiguïté de la position de cette langue, oscillant entre son statut "officiel » de langue étrangère et sa pratique réelle dans la société, obère les décisions la concernant. Les autres langues sont introduites, pour certaines, dès la première année du deuxième palier (la septième année fondamentale); c'est le cas de la langue anglaise, que certains partisans extrémistes de l'arabisation, connus pour leur hostilité à la langue française, auraient voulu voir supplanter cette dernière dans tous les paliers de l'enseignement d'une manière définitive.

\section{LANGUES DE SCOLARISATION, LANGUES DE L'ENSEIGNEMENT À L'UNIVERSITÉ ET MARCHÉ DU TRAVAIL}

D’importantes distorsions affectent la réforme de ce système scolaire pensé comme un champ clos, isolé et, surtout, en porte-à-faux avec les autres secteurs de la vie sociale du pays.

La première de ces distorsions touche le volet linguistique. L'école fondamentale est, depuis la fin des années quatre-vingt, complètement arabisée, avec

6. Consacrée par l'ordonnance du mois d'avril 1976 portant organisation de l'éducation et de la formation. 
une négligence avérée pour l'enseignement des autres langues et plus particulièrement de la langue française. D'où une rupture entre cet enseignement de base arabisé et un enseignement exclusivement francophone dans les filières scientifiques et technologiques à l'université. Alors que l'enseignement de la langue française va décliner d'une manière encore plus inquiétante au cours de la décennie de guerre civile ${ }^{7}$, les bacheliers sont sommés de se remettre au français s'ils veulent réussir leurs études dans ces filières. Quelle injustice pour ceux qui ont brillamment réussi au baccalauréat avec des moyennes élevées dans les matières scientifiques mais qui trébuchent à l'université pour cause de faiblesse dans la maîtrise de la langue française!

La deuxième distorsion relevée concerne l'énorme hiatus qui existe entre le monde de l'école et le monde économique, entre le monde scolaire fortement marqué par la langue arabe et le monde du travail qui fonctionne majoritairement en français, toujours en position dominante et qui bénéficie, face à l'anglais, d'être la véritable troisième langue pratiquée par les locuteurs algériens ${ }^{8}$. Deux mondes coexistent en Algérie, obéissant à deux logiques opposées : une logique identitaire, travaillée par l'idéologie nationaliste chère aux élites conservatrices, et une logique économique pragmatique, prônée par les élites dites modernistes. Ces deux logiques sont souvent en décalage avec les convictions profondément conservatrices de la majorité du peuple algérien. Ce clivage peut coïncider avec le clivage linguistique entre arabisants et francisants mais sans que cela soit systématique, car la variété des stratégies et des comportements des divers groupes sociaux semble surtout répondre à des intérêts individuels et collectifs de promotion sociale et de profit économique. Ce sont donc des familles, des parents appartenant à des milieux divers, qui vont développer des résistances au discours dominant en matière d'éducation, en mettant à mal très tôt le monopole exercé d'une manière autoritaire par l'État.

\section{Diversité des RÉsistances À L'ENCONTRE DE L'ÉCOLE PUBLIQUE ALGÉRIENNE}

Dès les premières années de l'indépendance, confortés par la présence des institutions scolaires françaises, beaucoup de parents d'élèves algériens ont continué à inscrire leurs enfants dans ces institutions. À Alger, le lycée Descartes va accueillir jusqu'à la fin des années quatre-vingt des centaines d'élèves algériens. Enfants de diplomates, de familles aisées ou ayant débuté leur scolarité dans un établissement français à l'étranger durant la guerre de libération, tous vont bénéficier d'un enseignement francophone avec des cours de langue arabe dispensés par des enseignants arabes ou arabisants de qualité. Dans le même

7. Les tenants de l'islamisme violent avait décrété illicite l'enseignement de cette langue.

8. Le choix d'enseigner en anglais fait par des instituts de formation supérieure spécialisée ne constitue pas - ou pas encore - une réelle menace pour le français. 
temps, et alors que le système algérien vit sous le régime des "tiers ", les enfants issus des classes sociales favorisées se retrouvent dans les classes bilingues. Cette discrimination pédagogique se colore d'une discrimination linguistique contribuant à dévaloriser d'une manière durable l'image de la langue arabe dans toute la population et à valoriser en revanche celle de la langue française, perçue comme étant celle de la modernité et de la réussite sociale.

L'arabisation complète de l'école, l'hiatus avec l'enseignement supérieur, la crise sociale qui va secouer le pays à la fin de cette troisième décennie de l'indépendance vont accélérer le mouvement de défiance envers l'école publique. Il faut rappeler que dès le milieu des années soixante-dix, l'État a décrété son monopole sur la formation et l'éducation, en fermant les institutions scolaires religieuses chrétiennes et en fondant dans le public, en 1976, le système d'enseignement qui dépendait du ministère des Affaires religieuses. Aux côtés des structures dépendant du système éducatif français, qui continuent à accueillir un nombre assez important d'enfants algériens, l'école égyptienne va avoir les faveurs de familles algériennes "fâchées » avec l'école de leur pays et de plus en plus convaincues qu'elle est devenue l'otage d'une idéologie nationaliste conservatrice. Dès le début des années quatre-vingt, des grèves secouent les lycées et les lycéens - comble de l'ironie - demandent de " jeter l'histoire à la poubelle ». La chute du prix du pétrole va accentuer les effets de la crise sociale, ce qui contribue à disqualifier le modèle algérien de développement, fondé sur le principe de la distribution de la rente et animé par une idéologie socialisante faussement égalitariste. L'école ne peut plus servir d'ascenseur social et exclut chaque année des centaines de jeunes sans qualification, qui n'ont plus alors pour perspective que le chômage, la délinquance ou l'absolu du discours religieux. $\mathrm{Au}$ même moment, à l'université, les mouvements islamistes de plus en plus présents se heurtent aux mouvements de gauche et plus particulièrement au Mouvement culturel berbère (MCB) qui lutte pour la reconnaissance de la langue et de la culture berbères. Mais l'alternative des écoles françaises va être définitivement bloquée avec la fermeture du lycée Descartes aux élèves algériens en septembre 1988 et celle de toutes les structures scolaires étrangères avec l'éclatement de la guerre civile. Ces événements successifs vont accélérer les initiatives privées, individuelles ou collectives, pour organiser des alternatives scolaires en dehors de l'enseignement public d'État. Les Algériens vont alors profiter de l'ouverture politique qu'a connue le pays après les sanglants événements du 5 octobre $1988^{9}$. La chute du régime du parti unique et l'instauration du multipartisme vont leur ouvrir le champ des possibles. La liberté d'entreprendre sur le plan économique et celle de créer des associations sont consacrées par la nouvelle Constitution de 1989. Ces deux brèches vont leur permettre de se

9. Le 5 octobre 1988 en Algérie a été marqué par des manifestations sporadiques et non contrôlées dans plusieurs villes du pays. L'armée algérienne est sortie de sa réserve pour contrôler la situation. La crise a duré plusieurs jours, les villes les plus touchées étant Alger, Annaba, Oran, Constantine, Tizi Ouzou, Béjaia. (Source : Wikipédia) (NdIR) 
constituer en associations de parents jouissant d'un registre de commerce et de créer des écoles privées, essentiellement des écoles primaires ou des jardins d'enfants dans un premier temps, puis des collèges et enfin des lycées. Ces structures clandestines tolérées mais non reconnues sont financées par des fonds privés levés par les parents des élèves et sont payantes, alors même que le droit à l'instruction bénéficie de la gratuité inscrite dans les textes fondamentaux de la République. Par ailleurs, nombre de ces écoles font le choix de suivre les programmes français et font, de ce fait, le pari d'un enseignement exclusivement francophone. Nous ne pensons pas qu'il faille réduire cette attitude à la seule insécurité linguistique et à l'hostilité à l'arabisation comme le suggère Mounira Cheriet (Cheriet, 2014). Le malaise est plus profond. Il est social, politique et identitaire. C'est une véritable remise en cause de tout ce qui a fait l'Algérie de l'indépendance, avec ses promesses de progrès et de développement. Il faut reconnaître que la défiance envers l'école publique trouve sa justification majeure dans le fait qu'elle a pu constituer un vivier pour le recrutement des sympathisants du FIS ${ }^{10}$ ou même d'une grande partie de ses militants convaincus, qui vont verser dans la violence. Paradoxalement, alors même que des millions d'enfants et des milliers d'enseignants et surtout d'enseignantes bravaient, au péril de leur vie, l'interdit du Groupe islamique armé (GIA) en prenant tous les matins les chemins de l'école, cette école est vilipendée parce qu'elle aurait "formé des monstres ». C'est dans ce climat de terreur et de grande violence que les écoles privées ont été créées $^{11}$. Le paysage scolaire qui se dessine alors est complètement hétérogène. Pour la petite enfance, l'offre se diversifie entre les structures publiques, qui n'arrivent pas à répondre à la demande, et les jardins d'enfants privés appliquant des tarifs prohibitifs pour la majorité des familles; l'enseignement traditionnel religieux se revivifie en ouvrant des classes dans les mosquées ou en rouvrant les anciens kouttab ${ }^{12}$ aux enfants entre 3 et 6 ans. L'hétérogénéité linguistique est de mise aussi ${ }^{13}$, clivant encore davantage la société. Aux côtés des écoles privées exclusivement francophones, d'autres moins nombreuses font le choix du bilinguisme en soumettant leurs élèves à un double enseignement en arabe et en français dans la même journée ${ }^{14}$. C'est donc dans une grande instabilité institutionnelle, sociale, économique et politique que l’Algérie meurtrie entre dans la première décennie du XXI ${ }^{\mathrm{e}}$ siècle.

10. Le Front islamique du salut (FIS), principal parti islamiste et acteur de la tragédie des années quatre-vingt-dix. 11. Actuellement, il n'existe que 292 établissements regroupant près de 60000 élèves. Cela peut paraître infime mais la dimension symbolique et politique de cette création est plus importante que les données chiffrées.

12. Écoles traditionnelles exclusivement consacrées à l'apprentissage du Coran et à quelques rudiments d'arabe.

13. Dans les structures publiques, c'est l'arabe qui devrait être majoritairement utilisé mais, en réalité, l'arabe dialectal et le français y ont une large place et beaucoup de jardins d'enfants privés font la part belle au français. Enfin, les kuttab sont exclusivement arabophones.

14. Nous regrettons que cette expérience de bilinguisme scolaire n'ait pas suscité l'intérêt des chercheurs jusqu'à nos jours. En réalité, c'est toute l'expérience de l'enseignement privé qui n'a pas encore fait l'objet de recherches sérieuses et documentées, comme si elle restait marquée du sceau du tabou et de l'anathème. 


\section{UNE RÉFORME DE L’ÉCOLE TIMIDE FACE AU DÉVELOPPEMENT DU MARCHÉ DES LANGUES}

Élu en 1999, le président Bouteflika entame son premier mandat en mettant en chantier un certain nombre de réformes dont celle, très attendue par toute la société, de l'École. La Commission de réforme du système éducatif (CRSE), composée de 160 membres aux sensibilités politiques contrastées, est installée le 13 mai 2000 et va siéger en sessions, telle un mini parlement. Après une année de travail, elle finalise un rapport remis au Président mais jamais publié. Ce rapport préconisait un certain nombre de mesures susceptibles de transformer radicalement le fonctionnement du système éducatif algérien. En revenant à l'architecture de l'école en trois degrés, primaire, moyen et secondaire, elle rompt définitivement avec l'esprit de l'École fondamentale. Elle propose d'avancer l'introduction de la langue française dès la deuxième année primaire ${ }^{15}$, d'élargir le préscolaire à tout le territoire (objectif non encore atteint en 2015) et recommande l'ouverture de l'école sur le monde et les innovations scientifiques en l'inscrivant dans l'évolution de la connaissance en matière d'éducation. Ces recommandations sont mises en œuvre par la réforme des années 2000, qui voit les lois d'orientation de 2003 et 2008 modifier d'une manière radicale l'ordonnance de 1976. La Commission échoue en revanche à faire appliquer sa recommandation d'enseignement bilingue de certaines matières au collège et au lycée.

Sans remettre en question les grands principes de démocratisation, de gratuité de l'instruction et de position dominante de la langue arabe en tant que langue de scolarisation, la mise œuvre de la réforme en 2003 va viser à recentrer les objectifs de l'école en adoptant les approches par compétences et à remettre, de ce fait, l'élève au centre des préoccupations des pédagogues. Elle va régler la question des écoles privées en leur donnant une couverture juridique. En 2005, le texte portant organisation de l'enseignement privé est promulgué. Ces écoles sont soumises à certaines conditions, dont la plus importante est d'appliquer les instructions et programmes nationaux pour être agréées. Un certain nombre d'entre elles vont continuer à donner à la langue française une position particulière en tant que langue d'enseignement, au risque de s'écarter des directives officielles, mais le décret remet les pendules à l'heure en intégrant ces écoles dans le giron de l'État.

La frilosité affichée du pouvoir dans la gestion du plurilinguisme dans ses implications didactiques et sociétales perdure, malgré les constats de plus en plus alarmants concernant les performances de l'école en matière de maîtrise des langues (arabe et française surtout). Les distorsions en matière d'orientation, la faiblesse des programmes de formation des formateurs, les grèves cycliques

15. Introduite en 2003 en deuxième année mais, dès l'année suivante, en raison de la l'insuffisance quantitative et qualitative du corps enseignant, reportée à la troisième année primaire. 
des enseignants, les atermoiements du pouvoir face aux tenants du conservatisme continuent à entretenir la défiance de la société envers l'école publique et poussent des groupes entiers de la population à s'adresser, au prix de sacrifices financiers douloureux, aux autres institutions ${ }^{16}$.

Les écoles étrangères étant de retour dans le pays avec la paix retrouvée, l'école saoudienne voit affluer des centaines de familles pour inscrire leurs enfants et être en phase avec une certaine conception de la pratique religieuse, de l'éducation et de la vie qui serait plus proche de l'orthodoxie musulmane. D'autres familles, faisant le pari de la modernité, utilisent tous les moyens, quitte à se saigner aux quatre veines, pour permettre à leurs enfants d'accéder au lycée Alexandre Dumas ${ }^{17}$ et à l'école primaire française qui a rouvert ses portes depuis trois ans. Le clivage linguistique continue de marquer d'une manière durable la société et les stratégies des familles algériennes, tant le discriminant de la langue dominante reste prégnant. La langue arabe est défendue à la fois par les élites « arabistes » et les élites islamistes, en raison de son lien avec la religion pour les uns et avec l'appartenance à la nation arabe pour les autres. Ces deux composantes se rejoignent pour défendre l'option de l'arabisation, même si elles divergent d'une manière frontale sur le contenu à lui donner, avec un avantage évident, ces dernières années, aux élites religieuses. La langue française, lestée de son passif colonial au sein des couches jeunes de la population qui accèdent maintenant à l'université et au monde du travail, continue à jouir d'une image positive dans toute la société et n'est plus l'apanage des seules élites francophones. Sa connaissance et sa maîtrise sont perçues comme un atout déterminant pour l'accès aux filières nobles à l'université, pour entreprendre des études à l'étranger (en France mais aussi au Canada, au Québec plus exactement), pour obtenir un emploi gratifiant. Ces aspirations sont largement partagées par divers groupes sociaux, y compris ceux qui ne sont pas soupçonnés d'appartenir au fameux «Hizb frança ${ }^{18}$.

L'été 2015 a vu la question des langues de nouveau passionnément débattue. Le débat s'est déplacé cette fois sur la question de la prise en compte des « langues maternelles » dans les premières années de scolarisation, alors que l'introduction de l'enseignement du tamazight (appellation désormais consacrée du berbère) en 1995 en tant que langue a été la conséquence de luttes très dures, sans susciter un véritable débat dans la société, comme si la majorité des Algériens ne semblait pas concernée par cette mesure ${ }^{19}$. Par contre, dès qu'il s'est agi de la darija, terme générique pour désigner les parlers arabes, le tollé a été général,

16. Il faudrait aussi noter ici le grand engouement des parents pour les cours de soutien qui drainent des sommes colossales.

17. Qui a remplacé l'ancien lycée Descartes.

18. "Le parti de la France » : c'est ainsi que l'on nommait les élites francophones soupçonnées de francophilie rédhibitoire.

19. La société algérienne, tout en adhérant au combat pour la démocratie du MCB, n’a jamais vraiment fait de la cause du berbère une cause nationale. 
démontrant la prégnance des positions normatives concernant la langue arabe, positions qui ignorent l'idée même de la variation inhérente à toute langue et veulent imposer une vision a-historique de l'évolution linguistique. La recommandation va très vite passer à la trappe, les conditions de sa mise en ouvre n'étant pas réunies. Encore une preuve du caractère sensible du choix des langues d'enseignement, qui, cinq décennies après l'accès à l'indépendance, peine à trouver une solution définitive et à bénéficier d'un consensus accepté par la majorité des Algériens.

La question des langues de scolarisation ne peut être envisagée d'une manière isolée en l'enfermant dans le monde clos de l'école. Il est nécessaire de poser le préalable du projet social. Or ce projet manque, faute d'une assise démocratique qui permette à la société, dans son ensemble, de participer à son élaboration. Malmenée par les effets d'une modernisation autoritaire, idéologisée à outrance, enfermée dans une vision patrimoniale chauvine et exclusive de l'identité et, in fine, bousculée par des velléités de réforme hâtives, l'école publique algérienne se voit soumise aux pressions de la société qui, en développant diverses stratégies de contournement et d'évitement, lui manifeste une défiance renouvelée. La fracture politique entre les Algériens et leur État et la perte de confiance en la capacité de cet État à prendre en charge les aspirations de la population, à construire un système éducatif performant et en phase avec l'évolution du monde sont telles que nous voyons des groupes sociaux se positionner hors de leur société, ne partageant plus avec la majorité de leurs compatriotes l'idéal d'une nation unie dans la quête d'un avenir meilleur.

L'alternative que nous préconisons pour remédier à cette situation est à la fois simple et compliquée car elle nécessite un certain courage politique non seulement de l'État mais aussi des élites qui doivent sortir de leur frilosité et de la satisfaction de leurs propres intérêts afin d'imaginer une stratégie produite d'une façon démocratique pour :

- réaliser une révolution copernicienne dans l'enseignement de la langue arabe, qui doit être enseignée comme une langue vivante; mener une réflexion hardie et sans concession sur le rôle de cette langue dans l'apprentissage des autres disciplines en encourageant la traduction et une production scientifique de qualité ;

- mener en parallèle une réflexion tout aussi hardie sur l'enseignement de la langue française, qui la mette en phase avec sa place réelle dans la société ; en faire une langue d'enseignement de certaines matières dès le collège pour réduire l'effet de rupture avec l'enseignement supérieur d'une part et permettre d'autre part une meilleure adéquation entre le monde scolaire et le monde du travail. 
Enfin, il faut mener une autre réflexion hardie sur le rôle des langues premières de socialisation dans la scolarisation, tout en mettant en place les outils qui puissent donner au tamazight la possibilité de devenir réellement une langue nationale, un patrimoine partagé et assumé par tous les Algériens. Mais il faudra procéder au préalable à une refondation complète du système de formation des formateurs, car sans enseignants qualifiés et conscients des enjeux de leur mission, toute réforme est vouée à l'échec. Il s'agit de mettre en place les conditions d'une politique éducative qui rompe de manière définitive avec l'idéologie scolaire monolingue et mononormative et opte pour la tolérance et l'ouverture à toutes les compétences et à toutes les initiatives : une politique qui prenne en compte la diversité culturelle et linguistique du pays et les aspirations des Algériens à être des citoyens de ce monde, où la pluralité est la règle et non l'exception. C'est à ce prix qu'il sera possible d'édifier une école où la réconciliation des jeunes Algériens avec leur pays, leur histoire et leur récit national pourra être consacrée.

\section{BIBLIOGRAPHIE}

ASSELAH-RAHAL S., BLANCHET P. (2007) : Plurilinguisme et enseignement des langues en Algérie, Fernelmont, EME.

BENGUERNA M., KADRI A. (2001) : Mondialisation et enjeux linguistiques. Quelles langues pour le marché du travail en Algérie?, Alger, Casbah Éditions.

BLANCHET P., CLERC S., RISPAIL M. (2014) : «Réduire l'insécurité linguistique des élèves par une transposition didactique de la pluralité sociolinguistique », ELA, 175, Paris, Didier/Klincksiek.

CHERIET M. (2014): L'insécurité en Algérie: les imprévus d'une politique des langues dans l'enseignement, ELA, 175, Paris, Didier/Klincksiek.

DOURARI A. (2003) : Les malaises de la société algérienne, Alger, Casbah Éditions.

GUERID D. (2007) : L'exception algérienne. La modernisation à l'épreuve de la société, Alger, Casbah Éditions.

HADDAB M. (2014) : Dimensions du champ éducatif algérien. Analyses et évaluations, Alger, Arak Éditions.

MADI M. (éd.) (1998) : "L'école en débat ", Réflexions, n 2, Alger, Casbah Éditions.

PAVEAU M. A. (2008) : "Le parler des classes dominantes, objet linguistiquement incorrect? ", ELA, 150, Paris, Didier/Klincksieck.

TALEB IBRAHIMI K. (1995): Les Algériens et leur(s) langue(s), Éléments pour une approche, de la société algérienne, Dar El Hikma, Alger, réédité en 1997.

THIESSE A. M. (1999) : La création des identités nationales, Paris, Le Seuil. 
